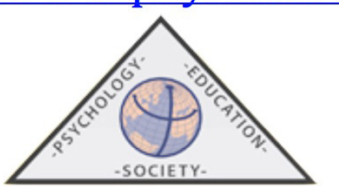

\title{
Prevalencia del sexting en adultos jóvenes universitarios: motivación y percepción del riesgo
}

\author{
Laura MONSALVE LORENTE y Enrique GARCÍA TORT
}

\author{
Universidad de Valencia
}

(Recibido el 11 de Mayo de 2020, Aceptado el 6 de Octubre de 2020)

RESUMEN: El sexting es un fenómeno en auge entre adolescentes y adultos jóvenes que consiste en enviar mensajes y/o archivos de carácter sexual a través de los smartphones y las redes sociales. Si bien no existe un claro consenso en su conceptualización, el sexting puede derivar en una serie de peligros como el cyberbullying o la sextorsión. Dicho fenómeno es clave a la hora de diseñar programas de educativos sexuales en adultos jóvenes. El presente estudio tiene como objetivo averiguar el grado de prevalencia del sexting en población adulta joven universitaria. Para ello se administró una serie de instrumentos a 288 estudiantes de entre 18 y 25 años de la Universitat de València, provenientes de los grados en Pedagogía, Maestro en Educación Infantil y Maestro en Educación Primaria. Los resultados indican que el sexting es una práctica poco frecuente en el día a día de los adultos jóvenes, aunque el 39,2\% declara haberlo practicado en alguna ocasión. A su vez, señalan como la valoración moral y la percepción del riesgo son dos factores relacionados con la frecuencia en la práctica de sexting. Aquellos sujetos que realizan una valoración moral negativa son menos propensos a realizar sexting y más propensos a valorar dicha práctica como arriesgada. Por otro lado, se hallaron otra serie de resultados que respaldan las aportaciones de la literatura científica, como la no diferencia entre hombres y mujeres respecto a la frecuencia o la mayor frecuencia en sujetos no heterosexuales respecto a los sujetos heterosexuales.

Palabras clave: Educación sexual, Adultos jóvenes, Prevalencia, Riesgo, Educación de adultos.

\section{Prevalence of Sexting in Young University Students: Motivation and Perception of Risk}

ABSTRACT: Sexting is a phenomenon on the rise among adolescents and young adults that consists
of sending messages and/or files of a sexual nature through smartphones and social networks. Although
there is no clear consensus in its conceptualization, bad management of sexting can lead to a number of
risks such as cyberbullying or sextortion. Sexting is a key phenomenon when designing sex education
programs in young adults. The present study aims to find out the degree of prevalence of sexting in the
young adult university population. To this end, a series of instruments were administered to 288 students
between 18 and 25 years of the University of Valencia, from the university degrees in Pedagogy, Teacher
in Early Childhood Education and Teacher in Primary Education. The results indicate that sexting is
a rare practice in the day to day of young adults, although $39.2 \%$ declare that they have practiced it
on occasion. In turn, they point out how moral assessment and risk perception are two factors related 
to the frequency of sexting. Those subjects who make a negative moral assessment are less likely to perform sexting and more likely to value such practice as risky. On the other hand, we found another series of results that support the contributions of scientific literature, such as the difference between men and women with respect to frequency or greater frequency in non-heterosexual subjects with respect to heterosexual subjects.

Keywords: Sexual Education, Young adults, Prevalence, Risk, Adult Programs.

Correspondencia: Laura Monsalve Lorente. Avda. Blasco Ibáñez, 13. 46010 Valencia. E-mail: laura. monsalve@uv.es

\section{Introducción}

\section{Conceptualización}

Internet, así como las redes sociales, han construido nuevas formas de socialización con un especial interés en los jóvenes (Barrense-Dias, Berchtold, Surís y Akre, 2017; Delevi y Weisskirch, 2013). Estas nuevas formas de comunicación cambian por completo el modelo de desarrollo y construcción social, generando nuevos retos en el desarrollo de aspectos psicosociales como la identidad, la socialización o la sexualidad (Barrense-Dias et al., 2017).

Entre los muchos fenómenos relacionados con las redes sociales y la comunicación, el sexting se hace especialmente relevante dada la falta de percepción de riesgo por parte de los adolescentes y las posibles consecuencias a nivel psicológico, jurídico y social (Molla-Esparza, Rodríguez-García, y López-González, 2016; Rickets, Maloney, Marcum, y Higgins, 2014; Ahern y Mechling, 2013; Martínez, 2013).

Entendiéndose en su definición más cercana a la sexualidad, el sexting es una práctica que implica compartir material de carácter sexual propio a través de las redes sociales (Gámez-Guadix, Santisteban, y Resett, 2017). De esta forma, es una conducta en auge entre adolescentes y jóvenes (Gerding, 2016), y la omnipresencia de los smartphones y las redes sociales han supuesto un incremento de esta práctica.

El sexting, a pesar de su actual interés educativo y social, sigue siendo un fenómeno sin una conceptualización consensuada en el ámbito científico. De facto, existen discrepancias entre los distintos autores a la hora de determinar sus características (Barrense-Dias et al., 2017). En la definición del concepto parecen existir puntos en común. Por ello, algunos autores proponen delimitar el sexting como el envío o publicación de contenido sexual a través del móvil y las redes sociales (Gámez-Guadix, Santisteban, y Resett, 2017). Sin embargo, las diversas investigaciones discrepan entorno a qué se considera como contenido sexual (Mercado, Pedraza y Martínez, 2016). Esta falta de consenso implica una seria barrera de cara al desarrollo de instrumentos estandarizados y programas de intervención (Molla-Esparza, Rodríguez-García y López-González, 2016), puesto que la identificación de las conductas y las variables relacionadas variarán significativamente en función de lo que se entienda por contenido sexual. A modo de ejemplo, la prevalencia de la práctica variará muy significativamente en función de si se considera en exclusiva aquel contenido sexual que sea propio del sujeto que lo practica, o si, por el contrario, también es considerado dentro de la definición el compartir contenido sexual de terceros. 
Recientes metaanálisis muestran componentes clave en la definición de sexting (Mercado et al., 2016). En términos generales, los autores relacionan dicho término con el envío de mensajes con carácter sexual a través del móvil. Sin embargo, la edad mínima de los participantes, los medios de difusión y la definición de contenido sexual son las tres principales variables que impiden un consenso en la definición (Mercado et al., 2016).

El concepto de sexting comenzó a aparecer en los medios científicos a raíz del suicidio de un estadounidense de 18 años tras haber sufrido un acoso sistemático por parte de sus compañeros en 2008 (Mercado et al., 2016). Este suceso alertó a la comunidad científica de los peligros que dicha práctica podría ocasionar. De este modo, una primera oleada de autores señaló que a la hora de catalogar el fenómeno era importante tener en cuenta que las imágenes enviadas fueran desnudos o semidesnudos tomadas por los propios sujetos (McLaughlin, 2010). Tras nuevas investigaciones y un aumento en la prevalencia, autores como Wolak y Finkelhor (2011) reflexionaron sobre la importancia de las características del emisor. De esta manera, se abrió el debate sobre si el sexting entre menores de edad estaba más cercano a un fenómeno sexual libre o a la pornografía infantil.

En adelante, nuevos autores proponen un acercamiento al concepto desde una perspectiva más aproximada a la expresión sexual, entrando en juego la intencionalidad del sujeto a la hora de catalogar un contenido como sexual o no sexual. Esta perspectiva incluye en su definición cualquier texto o archivo que no sea explícitamente sexual (McGraw, 2013), y rompe con la comparación entre sexting y pornografía. En suma, esta nueva oleada no sólo destaca que para hablar del fenómeno en cuestión debe haber un envío a través de los medios digitales de contenido sexual, sino que, además, dicho contenido no tendrá que ser propiamente explícito (Delevi y Weisskirch, 2013).

Esta perspectiva cobra especial relevancia dentro del debate sobre la conceptualización. Sobre todo, debido a que saca a la palestra la importancia de factores como la motivación, la actitud y la intencionalidad del autor o autora a la hora de practicarlo.

Por último, se observa otro tipo de corriente caracterizada por incluir la práctica del sexting como parte del desarrollo psicosocial del individuo. De este modo, el envío de imágenes sexuales puede considerarse como un fenómeno de "extimidad" (Narvaja y De Piero, 2016). Extimidad es un concepto de origen psicoanalista que implica exponer la propia intimidad o la vida privada de uno mismo en lo público de la red. Así pues, estos autores tratarían de buscar las causas de movimientos como el sexting o el vlogging en aspectos relacionados con el desarrollo de la individualidad en la sociedad Red. En adición, en un modo más radical, algunos autores incluso llegan a apostar por una relación entre el sexting y determinados trastornos de exhibicionismo recogidos en el Manual Diagnóstico DSM-5 (Mercado et al., 2016).

\section{Prevalencia del sexting en población adolescente y adulta joven}

La prevalencia del sexting en la población adolescente y adulta joven ha sido objetivo de estudio en muchas investigaciones (Mercado et al., 2016). Se entiende por población adolescente aquella que se encuentra entre los 12 y 17 años (Arnett, 2000). No obstante, la postergación de los roles adultos y otra serie de factores políticos, sociales y económicos han llevado los autores a distinguir una población posterior a la adolescencia: la adultez joven (Fierro-Arias y Moreno-Hernán- 
dez, 2007). De esta forma, los adultos jóvenes serán aquellos cuya edad se encuentre entre los 18 y 25 años (Fierro-Arias y Moreno-Hernández, 2007; Arnett, 2000). Si bien la mayoría de los estudios no se han realizado con población española, algunos de ellos han reportado prevalencias muy interesantes de hasta un 33\% de envío de mensajes o imágenes de desnudos entre jóvenes de 14 a 24 años (Ahern y Mechling, 2013).

Según Molina-Peral y Vecina-Navarro (2017) el ciberbullying y el sexting son realidades actuales, y sin la información e intervención a tiempo las secuelas psicológicas de la víctima pueden cronificarse y desencadenar diferentes tipos de trastornos psicológicos.

Continuando con el análisis de la prevalencia, puede observarse cómo existen diferencias en cuanto a la edad (Ahern y Mechling, 2013). La práctica suele ir relacionada con edades cercanas a la adultez joven, siendo menor la prevalencia en los sujetos de entre 12 y 15 años. Del mismo modo, los resultados parecen indicar que los jóvenes no envían mensajes en la misma medida que los reciben, existiendo prevalencias superiores en las recepciones (Ahern y Mechling, 2013).

Algunos estudios, como los que recoge la investigación de Agustina y Gómez-Duran (2016), trataron de analizar el género como variable dentro de la práctica de sexting, obteniendo resultados que marcan patrones distintos entre varones y mujeres; mientras que los hombres suelen actuar difundiendo material procedente de otro receptor, las mujeres suelen ser más emisoras y receptoras de material dentro de una relación (Henderson y Morgan, 2011; citado en Agustina y Gómez-Duran, 2016). Estos datos son corroborados a su vez por los estudios recogidos en otros países, que revelan cómo las mujeres son más receptivas a producir imágenes que los hombres (Ahern y Mechling, 2013). En cambio, en cuanto a prevalencia se refiere, otros estudios revelan que no existen diferencias significativas entre la prevalencia de sexting y el sexo del sujeto (Gámez-Guadix, et al., 2017).

En cuanto a la investigación del sexting en España, no se ha realizado un gran número de estudios (Agustina y Gómez-Duran, 2016). Aun con todo ello, destaca la ambiciosa investigación llevada a cabo por Gámez-Guadix, Almendros, Borrajo y Calvete (2015). En ella se analizó la prevalencia en una población española de adultos españoles entre 18 y 60 años $(\mathrm{N}=873)$. De ella se extrajo que no existían diferencias significativas en la prevalencia en función del sexo, aunque sí que se pudo observar como la práctica era más frecuente entre los adultos jóvenes y los sujetos no heterosexuales. En cuanto a la población adolescente, investigaciones posteriores confirmaron una vez más las diferencias en función de la edad (Gámez-Guadix et al., 2017): prevalencia del $13.5 \%$ desde los 12 a los 17 años, con una mayor prevalencia a los $17(36.1 \%)$ que a $\operatorname{los} 12$ años $(3.4 \%)$.

Sin embargo, tras observar los estudios llevados a cabo, se pueden determinar una serie de limitaciones en las investigaciones. En primer lugar, casi la totalidad de estudios consisten en análisis correlacionales a partir de cuestionarios elaborados ad hoc para la investigación (Mercado et al., 2016), existiendo pocos estudios de carácter cualitativo (Alonso-Ruido, et al., 2015). Este aspecto es comprensible si se tiene en cuenta la falta de un instrumento validado de referencia (Molla-Esparza, et al., 2016). Por otro lado, algunos autores observan anomalías en los resultados que podrían indicar ciertos sesgos por parte de los encuestados a la hora de responder a los instrumentos (Alonso-Ruido, et al., 2015). De esta manera, parece que los adolescentes 
poseen una especie de locus de control externo hacia la práctica: ninguno reconoce que lo hace, pero afirma con seguridad que las personas de su edad lo practican habitualmente.

\section{Los peligros del sexting}

Como fenómeno social y sexual, el sexting no queda ajeno a toda una serie de riesgos sociales, psicosexuales, e incluso penales (Agustina y Gómez-Duran, 2016; Martínez, 2013). Dada la facilidad para compartir y difundir contenidos multimedia a través de las redes sociales, dicho fenómeno puede llegar a ser una práctica sexual peligrosa si no se cuenta con la formación y educación sexual adecuada. En adición, algunas investigaciones han demostrado la relación entre el envío de imágenes íntimas y otras conductas de riesgo, aspecto que refuerza la concepción del sexting como conducta sexual de riesgo (Agustina y Gómez-Duran, 2016).

Aun con todo ello, difundir material sexual ajeno sin consentimiento supone cometer una serie de delitos contra la intimidad y el derecho al honor recogidos en el Código Penal español, que, en algunos casos, puede llegar a suponer penas de prisión de hasta 5 años de cárcel (Martínez, 2013). A su vez, si los participantes son menores de edad, también entraría en juego la ley de protección jurídica del menor (Martínez, 2013), agravando los supuestos delitos cometidos.

Por último, existe otro fenómeno delictivo vinculado al sexting que surge cuando la difusión a terceros del contenido sexual va ligada, a su vez, de una serie de chantajes hacia la víctima: la sextorsión (Guardiola, 2017). Esta forma de extorsión recibe su particularidad del origen del objeto de chantaje: imágenes o vídeos sexuales de la víctima. Así pues, cuando se produce el primer intercambio de imágenes, el chantajista coacciona a la víctima con difundir el contenido si no cumple con sus demandas. Demandas que, por lo general, suelen derivar en la toma de imágenes o vídeos cada vez más sexualizados. De este modo, la víctima cae en un bucle de extorsión donde las exigencias del chantajista son cada vez más frecuentes y exigentes (Guardiola, 2017).

\section{Educación afectivo-sexual y adultezjoven}

La educación afectivo-sexual es una asignatura pendiente en el sistema educativo español. Si se atiende al concepto de educación sexual que arrojan las últimas leyes educativas (LOMCE, 2013; LOE, 2006) se observa como comparan la educación sexual a la materia de reproducción y prevención de enfermedades de transmisión sexual. A nivel estatal, la dimensión afectiva de la educación sexual no cuenta con un marco legislativo de referencia, y queda a disposición de las Comunidades Autónomas suplir dicha carencia. Por otro lado, los colegios e institutos no cuentan con una asignatura marco para la educación afectivo-sexual, lo que obliga a impartir dichos conocimientos en el marco de las asignaturas de biología y cuerpo humano: de nuevo, una predominancia de la perspectiva biológica de la educación sexual.

En lo referente a la prevención de malas práxis del sexting, Gil-Llario y Ballester-Arnal (2017) sostienen que muchos adultos, profesores y figuras de autoridad no se encuentran preparados actualmente para enfrentarse a retos como el grooming o la sextorsión. A su vez, señalan la importancia de un trabajo estructural a todos los niveles de la comunidad educativa con el fin de controlar la distribución de las imágenes y evitar una posible evolución a casos de cyberbullying, acoso escolar y sextorsión (Gil-Llario y Ballester-Arnal, 2017). 
Como ya se destaca en un principio, numerosas investigaciones han recabado información sobre la prevalencia del sexting en adultos jóvenes (Ahern y Mechling, 2013). Aun así, son muy pocos los proyectos educativos destinados a dicha población. Si bien la población adolescente cuenta con fructuosas líneas de investigación y creación de programas (Calvo, 2016), la población adulta joven suele quedar fuera de objeto de intervención.

A pesar de este vacío en el desarrollo de proyectos, sí que se ha contado con ejemplos de programas desarrollados en especial a jóvenes adultos (Ahern y Mechling, 2013), los cuales sustentan su relevancia en las posibles consecuencias negativas de la práctica de sexting. Sin embargo, si se realiza una comparación con los proyectos en adolescentes, existen diferencias muy significativas en cuanto a financiación, planificación e impacto. Por ello, es menester insistir en la necesidad de desarrollo de programas educativos sexuales y afectivos en población adulta joven. Aunque la adolescencia sea una etapa clave en la educación sexual, el colectivo adulto también es una población objetivo de talleres, planes y programas de ámbito sexual; más aún si se tiene en cuenta la rápida intrusión de las redes sociales en la vida personal, profesional, académica y social de la llamada "generación Z" (Llaneza, 2017).

El presente estudio persigue los siguientes objetivos generales y específicos. Como objetivo general: averiguar el grado de prevalencia del sexting en población universitaria.

Y como objetivos específicos: evaluar el impacto que tiene el sexting dentro de la actividad sexual de los jóvenes adultos universitarios y universitarias, determinar las posibles diferencias en función del sexo, la edad, el estado sentimental y la orientación sexual, y esclarecer la relación existente entre la práctica y el riesgo percibido.

En consecuencia, las hipótesis de investigación desde las cuales parte este estudio son las siguientes:

- Aquellos sujetos que practican sexting tienen unos niveles de percepción de riesgo menor que aquellos que no.

- Los sujetos que practican sexting en pareja tienen una percepción de riesgo menor que aquellos sujetos que lo practican con terceros.

- Aquellos sujetos que tengan una valoración moral negativa del sexting tendrán una mayor percepción del riesgo que aquellos sujetos que tengan una valoración moral positiva.

- Aquellos sujetos que tengan una valoración moral negativa del sexting compartirán con menor frecuencia contenido de carácter sexual que aquellos que tengan una valoración moral positiva.

- A mayor edad, mayor práctica.

- A mayor edad, menor percepción de riesgo en la práctica.

- A mayor edad, mejor valoración moral de la práctica.

\section{Método}

El presente trabajo plantea una investigación de carácter cuantitativo basada en un estudio correlacional de las distintas variables a mesurar. A raíz de los objetivos y las hipótesis planteadas, se pretende obtener resultados mediante un instrumento de evaluación de las conductas de 
sexting. Dicho instrumento estará compuesto por cuestionarios previamente validados y será administrado a la población objetivo de manera presencial.

\section{Población}

En este estudio participaron 288 estudiantes de 18 a 25 años provenientes de los grados de Pedagogía (66,7\%), Maestro en Educación Primaria (25\%) y Maestro en Educación Infantil $(8,3 \%)$ de la Universitat de València $(\mathrm{N}=288)$.

Tabla 1. Frecuencia según Estudio de Procedencia

\begin{tabular}{lcccc}
\hline Titulación & Frecuencia & Porcentaje & Porcentaje válido & Porcentaje acumulado \\
\hline Pedagogía & 192 & 66,7 & 66,7 & 66,7 \\
Magisterio Primaria & 72 & 25,0 & 25,0 & 91,7 \\
Magisterio Infantil & 24 & 8,3 & 8,3 & 100,0 \\
Total & 288 & 100,0 & 100,0 & \\
\hline
\end{tabular}

Del mismo modo, en cuanto a género se aprecia un total de 55 hombres $(19,1 \%)$ frente a 233 mujeres $(80,9 \%)$. En cuanto a orientación sexual, 261 de los encuestados declararon ser heterosexuales $(90,6 \%), 11$ como heterosexuales $(3,8 \%), 14$ como bisexuales $(4,9 \%)$ y 2 como otras orientaciones no heterosexuales $(0.7 \%)$.

Tabla 2. Frecuencia según Sexo

\begin{tabular}{lcccc}
\hline Sexo & Frecuencia & Porcentaje & Porcentaje válido & Porcentaje acumulado \\
\hline Hombre & 55 & 19,1 & 19,1 & 19,1 \\
Mujer & 233 & 80,9 & 80,9 & 100,0 \\
Total & 288 & 100,0 & 100,0 & \\
\hline
\end{tabular}

Tabla 3. Frecuencia según Orientación Sexual

\begin{tabular}{lcccc}
\hline Orientación sexual & Frecuencia & Porcentaje & Porcentaje válido & Porcentaje acumulado \\
\hline Heterosexual & 261 & 90,6 & 90,6 & 90,6 \\
Homosexual & 11 & 3,8 & 3,8 & 94,4 \\
Bisexual & 14 & 4,9 & 4,9 & 99,3 \\
Otro & 2 & 0,7 & 0,7 & 100,0 \\
Total & 288 & 100,0 & 100,0 & \\
\hline
\end{tabular}

En cuanto al estado sentimental actual, 105 sujetos se encontraban solteros (36,5\%), 176 se encontraban en pareja estable $(61,1 \%), 5$ se encontraban en una relación abierta $(1,7 \%)$, y 2 declararon estar en otro tipo de relaciones sentimentales distintas a la soltería $(0.7 \%)$. 
El acceso a la muestra fue posible gracias a la colaboración desinteresada e indispensable de la Universitat de València y los estudiantes de los Grados de Pedagogía, Maestro en Educación Primaria y Maestro en Educación Infantil, quienes colaboraron de forma voluntaria y anónima en el estudio.

Tabla 4. Frecuencia según Estado Sentimental

\begin{tabular}{lcccc}
\hline Estado Sentimental & Frecuencia & Porcentaje & Porcentaje válido & Porcentaje acumulado \\
\hline Soltero/a & 108 & 36,5 & 36,5 & 36,5 \\
En pareja & 176 & 61,1 & 61,1 & 97,6 \\
Relación abierta & 5 & 1,7 & 1,7 & 99,3 \\
Otro & 2 & 0,7 & 0,7 & 100,0 \\
Total & 288 & 100,0 & 100,0 & \\
\hline
\end{tabular}

\section{Medidas e instrumentos}

Acorde con las hipótesis de investigación planteadas, en el presente estudio se aplicó una adaptación española de la Sexting Behaviors Scale (Dir, 2012), traducida y validada en población universitaria española por el equipo de Chacón-López, Romero, Aragón, y Caurcel (2016). Por otro lado, se diseñó tanto una escala de uso de redes sociales y aplicaciones móvil, como un breve cuestionario de percepción del riesgo en la práctica.

En cuanto a la adaptación española del Sexting Behaviors Scale, la Escala de Conductas sobre Sexting (ECS) fue validada por un estudio realizado con 985 universitarios españoles de entre 18 y 24 años de edad (Chacón-López, Romero, Aragón, y Caurcel, 2016). La ECS posee un total de 29 ítems en formato de respuesta de opción múltiple (escala tipo Lickert) de 5 grados, y cuenta con excelentes indicadores de fiabilidad y consistencia interna $(\alpha=.922)$. En cuanto a su estructura interna, la ECS posee 3 factores internos: disposición activa, participación real y expresión emocional.

El primero de ellos, la disposición activa hacia el sexting, reúne del ítem 10 al 25 de la escala, y recoge información relativa a la motivación y estilo en la práctica. El segundo, la participación real, reúne los 9 primeros ítems de la escala, y recoge información relativa a la frecuencia de envío o recepción de contenido sexual a través del teléfono móvil o Internet. Por último, la expresión emocional alberga los cuatro últimos ítems de la escala, y pretende analizar los sentimientos y la valoración moral subyacente a la práctica.

Con arreglo a corroborar las hipótesis de investigación planteadas en este estudio, se elaboró un instrumento adicional a la ECS: una escala de percepción del riesgo en la práctica.

Esta escala consta de 11 ítems tipo Likert con 5 grados, donde los sujetos deben marcar su grado de acuerdo con las distintas afirmaciones que se plantean. Dada la falta de instrumentos específicos para medir la percepción del riesgo en el sexting, se tuvo que construir dicha escala con el objetivo de obtener un valor de percepción de riesgo subjetivo que oscila entre 1 (práctica percibida como muy arriesgada) y 5 (práctica percibida como muy segura). 
En suma, en cuanto a las distintas variables a medir durante el estudio, y acorde a las hipótesis de investigación planteadas, fue necesario obtener la siguiente información:

- Datos sociodemográficos de interés (sexo, edad, estado sentimental, orientación sexual y titulación).

- Frecuencia y práctica ECS).

- Evaluación emocional y moral (ECS).

- Estilo y motivación en la práctica (ECS).

- Percepción del riesgo en la práctica.

\section{Validación del cuestionario}

El conjunto de escalas y cuestionarios, incluido la ECS, fueron administrados bajo un informe de validación a un grupo de 7 profesoras del grado de Pedagogía por la Universitat de València.

A modo de validación lógica, el comité de expertos realizó una labor de revisión y corrección de errores. También se revisaron aspectos relativos a la construcción de las escalas, como la formulación de los ítems, la congruencia ítem-objetivo o la independencia de sesgos. Dicho comité quedó formado, finalmente por 7 profesoras ( 5 del Departamento de Didáctica y Organización Escolar, y 2 del Departamento de Métodos de Investigación y Diagnóstico en Educación). Sus observaciones fueron integradas en la versión final de los instrumentos.

\section{Análisis}

La presente investigación recabó variables de naturaleza cuantitativa, las cuales fueron procesadas a través de una serie de análisis estadísticos.

Para corroborar las distintas hipótesis de investigación se analizó la relación entre las distintas variables de estudio a través de pruebas T para valores independientes y correlaciones (en función de la naturaleza de las variables a analizar).

A la hora de realizar los análisis estadísticos, se tomaron en cuenta las siguientes indicaciones en función de las exigencias de las hipótesis de investigación:

- En cuanto a la orientación sexual, se establecieron dos grupos, sujetos con orientación heterosexual, y sujetos con orientación no heterosexual (que incluye homosexualidad, bisexualidad y otras orientaciones sexuales).

- Para comparar grupos en función de la práctica o no práctica de sexting, se tuvo en cuenta la puntuación obtenida en el segundo factor de la ECS, frecuencia, que abarca los 9 primeros ítems de la escala. Aquellos sujetos cuya puntuación en dicho factor fue inferior a 1 quedaron clasificados como 'no practicantes', y aquellos sujetos cuya puntuación fue igual o superior a 1 quedaron clasificados como 'practicantes'.

- A la hora de calcular la frecuencia de envío y recepción de contenido sexual, se recalcularon dos nuevas variables a partir de las puntuaciones medias de los ítems 6, 7, 8 y 9 (para envío) y 1, 2, 3, 4 y 5 (para recepción) de la ECS.

- A la hora de analizar la relación entre la variable 'percepción del riesgo' y el resto de las variables de interés, se obtuvieron numerosas correlaciones negativas. Para facilitar el análisis y la comprensión de los resultados se invirtieron las puntuaciones, de modo que 
la escala obtiene valores del 1 (práctica percibida como totalmente segura) al 5 (práctica percibida como muy arriesgada).

Todos los análisis y procesamientos estadísticos se realizaron a través del programa informático IBM SPSS Estadistic Base, en su versión 22.

\section{Resultados}

Tras el análisis del conjunto de datos obtenidos, se han obtenido los siguientes resultados.

\section{Frecuencia y medias generales}

En cuanto a la frecuencia general de participación (Tabla 5), se observa una media general de $0.92(\mathrm{~N}=288)$. Partiendo de una escala tipo Likert con 5 grados de libertad (con valores del 1 al 5), la media general obtuvo una puntuación inferior al 1, lo que indica un nivel de práctica bajo. Del mismo modo, si se analiza por separado la frecuencia para el envío y la frecuencia para la recepción (Tabla 5), se observa diferencias entre las medias; siendo de un nivel medio-bajo en la recepción $(1,20)$ y de un nivel bajo en el envío $(0,57)$. En suma, los sujetos son más propensos a la recepción que al envío de contenido sexual.

Tabla 5. Medias Generales

\begin{tabular}{lccc}
\hline & N & Media & Desviación estándar \\
\hline Frecuencia & 288 & 0,9236 & 0,59253 \\
Valoración moral & 288 & 1,6817 & 0,89183 \\
Percepción riesgo & 288 & 3,1239 & 0,63596 \\
Envío & 288 & 0,5747 & 0,56585 \\
Recepción & 288 & 1,2028 & 0,70221 \\
\hline
\end{tabular}

En términos generales (Tabla 5), la valoración emocional y moral de la práctica obtuvo una puntuación media de 1,68, lo cual presupone una valoración neutra con tendencias a una valoración positiva. Se recuerda que la escala utilizada recogía valores entre el 0 (valoración moral positiva) y 4 (valoración moral negativa).

A su vez, se obtuvieron las medias generales de percepción del riesgo en la práctica. En una escala del 1 (práctica percibida como totalmente segura) al 5 (práctica percibida como muy arriesgada), se obtuvo una media general de 3,12. Ello indica, de nuevo, una valoración media del riesgo por parte de la población total.

Continuando con los análisis generales (Tabla 6), 175 sujetos declararon no practicar sexting $(60,8 \%)$ de manera frecuente, frente a 113 sujetos $(39,2 \%)$ que habían enviado, recibido e intercambiado contenido sexual a través de Internet o el móvil con, al menos, una mínima frecuencia. Del mismo modo, entre aquellos sujetos practicantes, $63(55,8 \%)$ lo practicaban con su pareja, mientras que los 50 sujetos restantes $(44,2 \%)$ lo practicaban con terceros (Tabla 7 ). 
Tabla 6. Sujetos Practicantes versus No Practicantes

\begin{tabular}{lcccc}
\hline Práctica & Frecuencia & Porcentaje & Porcentaje válido & Porcentaje acumulado \\
\hline No practicante & 175 & 60,8 & 60,8 & 60,8 \\
Practicante & 113 & 39,2 & 39,2 & 100,0 \\
Total & 288 & 100,0 & 100,0 & \\
\hline
\end{tabular}

Tabla 7. Práctica en Pareja versus Con Terceros

\begin{tabular}{lcccc}
\hline Práctica & Frecuencia & Porcentaje & Porcentaje válido & Porcentaje acumulado \\
\hline Terceros & 50 & 44,2 & 44,2 & 44,2 \\
En pareja & 63 & 55,8 & 55,8 & 100,0 \\
Total & 113 & 100,0 & 100,0 & \\
\hline
\end{tabular}

\section{Pruebas T}

A continuación, se presentan los resultados obtenidos en las distintas comparaciones de medias a través de pruebas $\mathrm{T}$.

La prueba T realizada para estudiar la relación entre la práctica y su percepción del riesgo revela una diferencia significativa $(\mathrm{p}<0,00)$ entre las medias de los sujetos no practicantes $(3,28)$ y los sujetos practicantes $(2,88)$; siendo que aquellos sujetos que practican sexting tienen una percepción del riesgo menor que aquellos que no lo practican.

La prueba $\mathrm{T}$ realizada para estudiar la relación entre la práctica en pareja y la percepción del riesgo revela una diferencia significativa $(\mathrm{p}<0,00)$ entre las medias de los sujetos que practican sexting con terceros $(3,25)$ y los sujetos que lo practican en pareja $(2,78)$; resultando que aquellos sujetos que practican sexting en pareja tienen una percepción del riesgo menor que aquellos que lo practican con terceras personas.

\section{Correlaciones}

En adición, se presentan los resultados obtenidos en las distintas correlaciones llevadas a cabo entre las variables de naturaleza cuantitativa.

Los resultados indican que existe una relación significativa $(\mathrm{p}<0,00)$ entre la valoración moral y la percepción del riesgo; con un valor de en la correlación de Pearson de 0,324. Esto indica que, a peor valoración moral, mayor percepción de riesgo en la práctica (Tabla 9). Del mismo modo, los resultados indican que existe una relación significativa $(\mathrm{p}<0,00)$ entre la valoración moral y la frecuencia en la práctica; con un valor en la Correlación de Pearson de -0.273. Ello indica que, a mejor valoración moral, mayor es la frecuencia en la práctica (Tabla 9) 
Tabla 8. Correlación Valoración Moral/Percepción del Riesgo

\begin{tabular}{llcc}
\hline & & Valoración moral & Percepción del riesgo \\
\hline Valoración moral & Correlación Pearson & 1 & 0,324 \\
& Sig. (bilateral) & & 0,000 \\
& $\mathrm{~N}$ & 288 & 288 \\
Percepción del riesgo & Correlación Pearson & 0,324 & 1 \\
& Sig. (bilateral) & 0,000 & 288 \\
\hline
\end{tabular}

Tabla 9. Correlación Valoración Moral/Frecuencia

\begin{tabular}{llcc}
\hline & & Valoración moral & Percepción del riesgo \\
\hline Valoración moral & Correlación Pearson & 1 & $-0,273$ \\
& Sig. (bilateral) & & 0,000 \\
& $\mathrm{~N}$ & 288 & 288 \\
Frecuencia & Correlación Pearson & $-0,273$ & 1 \\
& Sig. (bilateral) & 0,000 & 288 \\
\hline
\end{tabular}

Por último, las correlaciones realizadas entre la edad y las variables de valoración moral, percepción del riesgo y frecuencia no han mostrado una relación significativa (Tablas 10, $11 \mathrm{y}$ 12). Esto podría indicar que, en el rango de edad de 18 a 25 años, la edad no es un factor influyente en la valoración moral $(\mathrm{p}<0,615)$, la percepción del riesgo $(\mathrm{p}<0,818)$ o la frecuencia en la práctica $(\mathrm{p}<0,195)$.

Tabla 10. Correlación Edad/Valoración Moral

\begin{tabular}{llcc}
\hline & & Valoración moral & Percepción del riesgo \\
\hline Valoración moral & Correlación Pearson & 1 & 0,030 \\
& Sig. (bilateral) & & 0,615 \\
& $\mathrm{~N}$ & 288 & 288 \\
Edad & Correlación Pearson & 0,030 & 1 \\
& Sig. (bilateral) & 0,615 & 288
\end{tabular}


Tabla 11. Correlación Edad/Percepción del Riesgo

\begin{tabular}{llcc}
\hline & & Valoración moral & Percepción del riesgo \\
\hline Edad & Correlación Pearson & 1 & $-0,014$ \\
& Sig. (bilateral) & & 0,818 \\
& $\mathrm{~N}$ & 288 & 288 \\
Percepción del riesgo & Correlación Pearson & $-0,014$ & 1 \\
& Sig. (bilateral) & 0,818 & 288 \\
& $\mathrm{~N}$ & 288 & 2 \\
\hline
\end{tabular}

Tabla 12. Correlación Edad/Frecuencia

\begin{tabular}{llcc}
\hline & & Valoración moral & Percepción del riesgo \\
\hline Edad & Correlación Pearson & 1 & 0,077 \\
& Sig. (bilateral) & & 0,195 \\
& $\mathrm{~N}$ & 288 & 288 \\
Frecuencia & Correlación Pearson & 0,077 & 1 \\
& Sig. (bilateral) & 0,195 & 288 \\
\hline
\end{tabular}

\section{Discusión}

En referencia a la práctica del sexting, los resultados obtenidos han permitido reflejar la importancia de la valoración emocional/moral y la percepción de riesgo.

Los resultados obtenidos indican que la frecuencia con la que los adultos jóvenes envían o reciben contenido sexual a través del móvil y/o las redes sociales mantienen una relación con la valoración moral y cuánto riesgo perciben en dicha práctica. De este modo, aquellos sujetos practicantes parecen valorar la práctica como más segura respecto de aquellos que no lo practican. Lo mismo ocurre con la valoración moral, siendo que aquellos sujetos que participan de este fenómeno con más frecuencia tienden a ver la práctica como más positiva a nivel moral/ emocional. Estos resultados señalan en la misma dirección de la literatura científica, donde el sexting se vincula a una falta de percepción del riesgo y las posibles consecuencias (Molla-Esparza, Rodríguez-García, y López-González, 2016; Rickets, Maloney, Marcum, y Higgins, 2014; Ahern y Mechling, 2013; Martínez, 2013).

Por otro lado, si bien ciertos estudios llevados a cabo en población adolescente mostraban prevalencias mayores conforme se incrementaba la edad (Ahern y Mechling, 2013), nuestro estudio muestra resultados contrarios para con la población joven adulta. La diferencia entre las medias de las distintas edades evaluadas no es significativa, por lo que en población adulta joven no parece existir una diferencia en cuanto a la frecuencia, la valoración moral o la percepción de riesgo a medida que aumenta la edad. 
En cuanto a la frecuencia general de participación en población universitaria se obtiene un nivel general de participación bajo. Sin embargo, si se analiza por separado la frecuencia para el envío y la frecuencia para la recepción, se observa diferencias entre las medias; siendo de un nivel medio-bajo en la recepción y de un nivel bajo en el envío. Este peculiar estilo a la hora de declarar mayores tasas de recepción que de emisión coincide con los sesgos observados por algunos investigadores en población adolescente (Alonso-Ruido, et al., 2015), lo cual podría indicar un posible locus de control externo a la hora de declarar la participación a través de cuestionarios. En cuanto a los datos de prevalencia, nuestro estudio a determinado que el 39,2\% de los adultos jóvenes encuestados lo han practicado alguna vez, bien sea a través del envío, la recepción o el intercambio de mensajes u archivos de carácter sexual.

En conclusión, el sexting es una práctica sexual que continúa siendo poco frecuente en la población adulta joven universitaria. Sin embargo, son pocos los adultos jóvenes que no hayan enviado, recibido, publicado o intercambiado contenido sexual a través del móvil alguna vez. Por tanto, y dada la relación que este estudio ha conseguido demostrar entre la percepción de riesgo y la frecuencia en la práctica, es importante generar nuevos campos de estudio que mejoren la formación en educación sexual y alerten de los posibles peligros que entraña dicha práctica.

\section{Limitaciones de la investigación}

Dadas las características y distribución de la muestra, el presente estudio muestra una serie de limitaciones ha tener en cuenta.

Esencialmente, las limitaciones a la hora de obtener una muestra representativa han derivado en una escasa representatividad del sexo masculino y orientaciones sexuales no heterosexuales. Por ende, y para evitar ofrecer resultados sesgados, se han eliminado del estudio todos los análisis y resultados relacionados con el Grado de procedencia, el sexo o la orientación sexual.

\section{Futuras líneas de investigación}

El presente estudio podrá continuar a través de futuras líneas de investigación donde, a raíz de los resultados obtenidos, se podrá continuar con el análisis de la prevalencia, las consecuencias, la percepción del riesgo y la motivación de los adultos jóvenes con respecto a las nuevas prácticas sexuales en la red. De esta forma, es menester realizar futuros estudios con una muestra representativa en cuanto a género, orientación sexual y Grado de procedencia que permita realizar análisis más profundos y generalizables.

\section{Referencias}

Agustina, J. y Gómez-Duran, E. (2016). Factores de riesgo asociados al sexting como umbral de diversas formas de victimización: estudio de factores correlacionados con el sexting en una muestra universitaria. Revista de Internet, Derecho y Política, 22, pp.32-58.

Ahern, N. y Mechling, B. (2013). Sexting: serious problems for youth. Journal of psychosocial nursing, 51(7), pp.22-30. DOI: 10.3928/02793695-20130503-02

Alonso-Ruido, P., Rodríguez-Castro, Y., Pérez-André, C. y Magalhães, M. (2015). Estudio cualitativo en un grupo de estudiantes ourensanos/as sobre el fenómeno del Sexting. Revista de Estudios e Investigación en Psicología y Educación, 0(13), pp.58-62. DOI: 10.17979/reipe.2015.0.13.319 
Arnet, J.J. (2000). Emerging adulthood: a theory of development from the late teens through the twenties. American Psychologist, 55(5), pp.469-480.

Barrense-Dias, Y., Berchtold, A., Surís, J. y Akre, C. (2017). Sexting and the definition issue. Journal of adolescent health, 61, pp.544-554. http://dx.doi.org/10.1016/j.jadohealth.2017.05.009

Chacón-López, H., Romero, J., Aragón, Y. y Caurcel, M. (2016). Construcción y validación de la Escala de Conductas sobre el Sexting (ECS). Revista Española de Orientación y Psicopedagogía, 27(2), pp.99-115.

Calvo, S. (2016). La práctica del sexting como estrategia de comunicación efectiva en parejas jóvenes: implicaciones para la intervención socioeducativa desde el marco de educación sexual. En: R. Roig-Vila, ed., Tecnología, innovación e investigación en los procesos de enseñanza-aprendizaje, 1st ed. Barcelona, España: Ediciones OCTAEDRO, S.L., pp.183-190.

Delevi, R. y Weisskirch, R. (2013). Personality factors as predictors of sexting. Computers in Human Behavior, 29, pp.2589-2594. http://dx.doi.org/10.1016/j.chb.2013.06.003

Dir, A.L. (2012). Understanding sexting behaviors, sexting expectancies, and the role of impulsivity in sexting behaviors. Doctorado. Purdue University. Recuperado de https://bit.ly/2P1AMPZ

Fierro-Arias, D. y Moreno-Hernández, A. (2007). Emerging adulthood in Mexican and Spanish youth. Theories and realities. Journal of Adolescent Research, 22, pp.476-503.

Gámez-Guadix, M., Almendros, C., Borrajo, E., Calvete, E. (2015). Prevalence and Association of Sexting and Online Sexual Victimization Among Spanish Adults. Sexuality Research and Social Policy, 12, pp. 145-154.

Gámez-Guadix, M., de Santisteban, P. y Resett, S. (2017). Sexting among Spanish adolescents: prevalence and personality profiles. Psicothema, 29(1), pp.29-34. http://dx.doi.org/10.1007/s13178-015-01869

Gavidia, V. (coord.) (2017). Los ocho ámbitos de la educación para la salud en la escuela. $1^{\mathrm{a}}$ ed. Valencia, España: Tirant lo Blanch, (pp. 211-226). DOI: 10.7334/psicothema2016.222

Gerding, A. (2016). Adolescent sexting: an examination of the psychosocial contributions to the creations and sharing of sexual images. Doctorado. Universidad de Missouri.

Gil-Llario, M.D. y Ballester-Arnal, R. (2017). El sexting: un nuevo reto para la educación sexual en la escuela. En V. Gavidia (Coord.), Los ocho ámbitos de la Educación para la Salud en la Escuela (pp.211-226). Valencia, España: Tirant Humanidades.

Guardiola, M. (2017). Menores y nuevas tecnologías: los nuevos retos en el sector legal en España. La ley Derecho de Familia: Revista jurídica sobre familia y menores, 14, pp.3-16.

Ley Orgánica 2/2006 de Educación. Boletín Oficial del Estado, España, 3 de mayo de 2006.

Ley Orgánica 8/2013 para la mejora de la calidad educativa. Boletín oficial del Estado, España, 9 de diciembre de 2013.

Llaneza, P. (2017). La Generación Z: incógnitos y privados. Revista de Estudios de Juventud, 114, pp.143-156.

Martínez, J. (2013). La difusión de sexting sin consentimiento del protagonista: un análisis jurídico. Derecom, 12, pp.1-16.

McGraw, J. (2013). Sexting unleashed: the social phenomenon according. Máster. Portland State University.

McLaughlin, J. H. (2010). Crime and Punishment: Teen Sexting in Context. Florida Coastal School of Law. Penn State Law Review, 135. Recuperado de https://bit.ly/2DSLrvB

Mercado, C. T., Pedraza, F. J. y Martínez, K. I. (2016). Sexting: su definición, factores de riesgo y consecuencias. Revista sobre la infancia y la adolescencia, 10, pp.1-18. http://dx.doi.org/10.4995/ reinad.2016.3934 
Molina-Peral, J.A. y Vecina-Navarro, P. (2017). Bullying, ciberbullying y sexting: ¿cómo actuar ante una situación de acoso? (2 ${ }^{\mathrm{a}}$ ed.). Madrid, España: Pirámide.

Molla-Esparza, C., Rodríguez-García, L. y López-González, E. (2016). Difficulties and solutions in the construction of an instrument to detect teen sexting. Comunicación presentada en el VII European Congress of Metodology, Mallorca, España.

Molla-Esparza, C., Rodríguez-García, L. y López-González, E. (2016). Sexting en adolescentes ¿Conscientes del peligro? Comunicación presentada en el XVI Congreso Nacional y VII Congreso Iberoamericano de Pedagogía, Madrid, España.

Narvaja, M.E. y De Piero, J.L. (2016). Prácticas juveniles éxtimas: sexting y vlogging. Aposta, Revista de Ciencias Sociales, 69, pp.239-270.

Rickets, M., Maloney, C., Marcum, C. y Higgins, G. (2014). The effect on Internet related problems on the sexting behaviors of juveniles. American Journal of Criminal Justice, 40, pp.270-284.

Wolak, J. y Finkelhor, D. (2011). Sexting: A typology. Crimes Against Children Research Center. Recuperado de: https://bit.ly/2DDwflE 